\title{
A Bayesian approach to quantify the uncertainties in the determination of galaxy properties derived from spectral fits
}

\author{
Gladis Magris C. ${ }^{1}$, Cecilia Mateu ${ }^{1}$ and Gustavo Bruzual A. ${ }^{1,2}$ \\ ${ }^{1}$ Centro de Investigaciones de Astronomía (CIDA) \\ Apdo. Postal 264, Mérida 5101, Venezuela \\ email: magris, cmateu@cida.ve \\ ${ }^{2}$ Centro de Radioastronomía y Astrofísica, UNAM, Campus Morelia \\ Apartado Postal 372, 58090, Morelia, Michoacán, México \\ email: g.bruzual@crya.unam.mx
}

\begin{abstract}
We use a bayesian formalism to quantify the uncertainties in the determination of the luminous mass and age of the dominant stellar population in a galaxy obtained from simple spectral fits. The analysis is performed over a sample of synthetic spectra covering a wide range of star formation histories and seen at different ages and redshifts. Using the bayesian approach we can establish quantitatively the uncertainties in the parameters derived from these fits in a straightforward manner, which is not possible using some simple algorithms, e.g. GASPEX, a non-negative least-square fitting algorithm.
\end{abstract}

Keywords. methods: data analysis, methods: statistical, galaxies: stellar content

\section{Introduction}

SED fitting methods have proven successful in carefully reproducing the observed SEDs with a vast variety of galaxies. It is well known that, in spite of the success in the spectral fitting process, the recovered physical parameters suffer from uncertainties that must be evaluated and made publicly available to the final users of such information. Walcher et al. (2010) present a wide discussion on this topic, showing the main techniques used to fit SED models to observations of galaxies, including an analysis of the limitations and uncertainties behind this processes. Bayesian fitting, and bayesian analysis of the uncertainties, is currently a common way to investigate the accuracy to which physical parameters are derived from galaxy spectra. Kauffmann et al. (2003), Da Cunha et al. (2008), Acquaviva et al. (2011), Benitez (2000), Pirzkal et al. (2011) are some of the authors who report the accuracy of their results by means of this technique.

\section{Method}

Our test is based on Dinbas3D, a dynamical method, successfully implemented to fit the spectra of simple and composite stellar populations, which has been proved to recover with great accuracy the mass and ages of galaxies of a variety of star formation histories (Mateu 2009; Cabrera 2011). In Dinbas3D the model galaxy's SED is assumed to be a linear combination of three simple stellar population SEDs $f_{i}(\lambda)$, from the library of Charlot \& Bruzual (2007), of arbitrary ages $t_{i}$ :

$$
\sum_{i=1}^{3} a_{i} f_{i}(\lambda)
$$



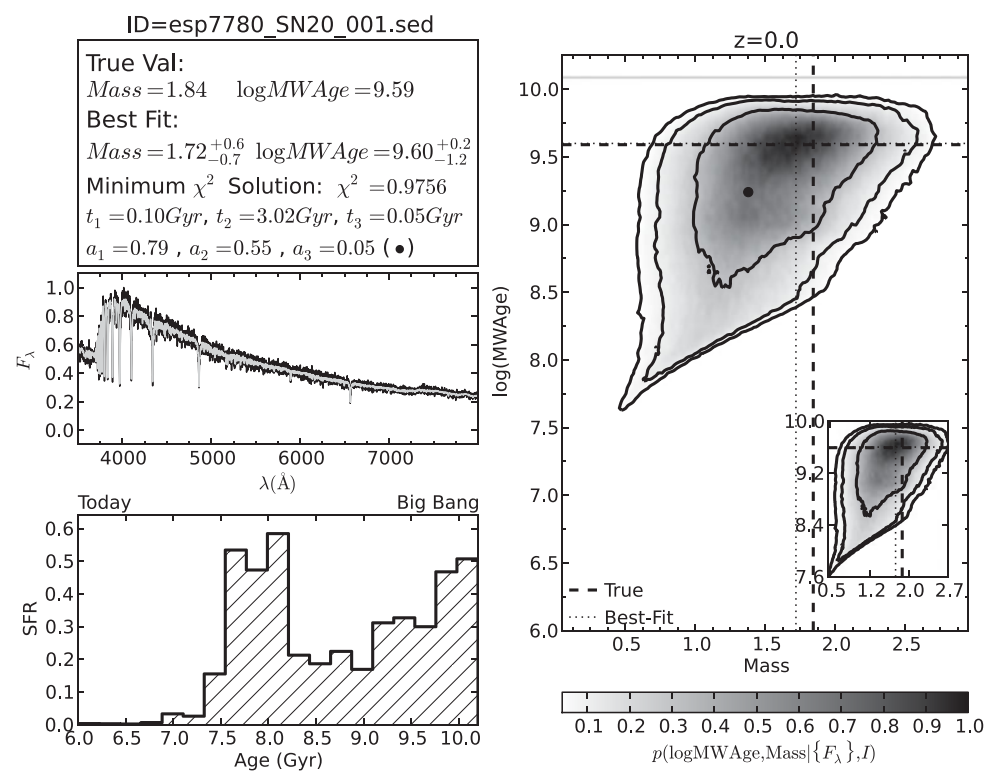

Figure 1. Right panel: 2D posterior pdf as a function of mass and mass-weighted age. Contours represent $1 \sigma, 2 \sigma$ and $3 \sigma$ confidence intervals. The black dot corresponds to the minimun $\chi^{2}$ model. Left Bottom: Derived star formation rate, as a function of age. Left Middle: Problem spectrum (black), minimun $\chi^{2}$ fit (grey). Left Top: Input and best fit parameter summary.

and the best fitting model is the model with the three pairs $a_{i}, t_{i}$ that minimizes the difference:

$$
\chi^{2}=\sum_{l}\left(F_{m o d}\left(\lambda_{l}\right)-F_{o b s}\left(\lambda_{l}\right)\right)^{2} / \sigma_{l}^{2}
$$

We use the Charlot \& Bruzual (2007) stellar population synthesis models to generate a library of 'mock galaxy' spectra. For $\mathrm{z}=0$, we choose a subset of the star formation rate histories suggested by Kauffmann et al.(2003), and for $z>0$, the SFR was set equal to $\exp (-t / \tau=1)$ or SSP for $z>1$. We add random gaussian noise corresponding to a constant $\mathrm{S} / \mathrm{N}$ ratio equal to 20 . In order to explore the robustness of the solutions, and to quantitatively report the uncertainties in the physical parameters (mass and age), we compute $10^{8}$ models with $a_{i}, t_{i}$ randomly drawn from a prior, Prior $(a, t)$, probability distribution function (pdf), which is uniform in $a_{i}$ and $\log \left(t_{i}\right)$. We then evaluate the posterior pdf $p\left(a, t \mid F_{o b s}\right)=\operatorname{Prior}(a, t) \exp \left(-\chi^{2} / 2\right)$ at each of these models and marginalize it to obtain the 2-D posterior $p\left(\right.$ Mass, $\left.M W A g e \mid F_{o b s}\right)$, which depends only on the total mass and the mass-weighted-age. The best-fitting solution is thus given by the maximum of $p\left(\right.$ Mass, MW Age $\left.\mid F_{\text {obs }}\right)$ and the mass and age uncertainties are given by the corresponding $1 \sigma$ confidence intervals.

\section{Conclusions}

In Figures 1 and 2 we synthesize the results of our spectral galaxy modelling. For each galaxy we show the $2 \mathrm{D}$ posterior pdf as a function of mass and mass-weighted age, the recovered star formation history, together with the numerical values of the best solution and the input parameters. We conclude that: 

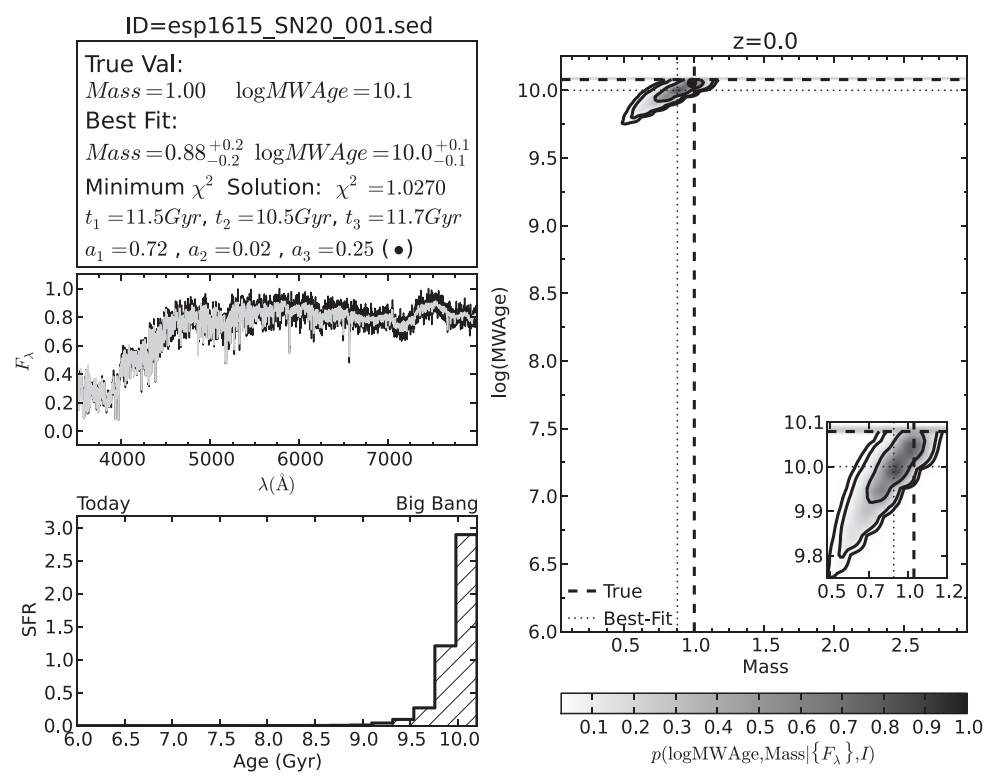

Figure 2. Same as figure 1

- For galaxies forming large amounts of stars at present or in the recent past, the uncertainty can be as large as a factor of 2 in age and a factor of 2.6 in mass. (Figure 1)

- Using a bayesian formalism we find that the mass and mean galaxy age can be estimated with a 10-15\% accuracy for quiescently star forming galaxies.

- The more exact and precise solutions are obtained for galaxies with pure old population (Figure 2)

- The uncertainty in the derived mass and, to a lesser extent, in age, increases with redshift.

- In general, the same results are obtained if we use more than 3 components in the fits

\section{Acknowledgements}

G. Bruzual acknowledges support for this work by the National Autonomous University of Mexico, through grant IA102311.

\section{References}

Acquaviva, V., Gawiser, E., \& Guaita L. 2011, ApJ, 737, 47

Benitez, N. 2000, ApJ, 536, 571

Cabrera, I. 2011, Bachelor thesis Universidad Central de Venezuela

Charlot, S. \& Bruzual, A. G. 2007, in preparation

Da Cunha, E., Charlot, S., \& Elbaz, D. 2008, MNRAS, 388, 1595

Kauffmann, G, Heckman, T. M., White, S. D. M. et al. 2003, MNRAS, 341, 33

Mateu, J. 2009, Rev. Mexicana AyA (CS) 164

Pirzkal, N., Rothberg, B., \& Nilsson, K. K. et al. 2011, ApJ, (submitted) arXiv: 1104.0054v1

Walcher, J., Groves, B., Budavári, T., \& Dale, D. 2010, ApESSS, 331, 1 